\title{
FROM PARTICLE SIZE ANALYSIS (PSA 1970) TO PARTICULATE SYSTEMS ANALYSIS (PSA 2003) ${ }^{\dagger}$
}

\author{
J. DODDS ${ }^{1, *}$, G. RASTEIRO ${ }^{2}$, B. SCARLETT ${ }^{3}$, \\ R. WEICHERT ${ }^{4}$ and R. WILLIAMS ${ }^{5}$ \\ EFCE WP Characterisation of Particulate Solids: ${ }^{1}$ Ecole des Mines d'Albi, France \\ ${ }^{2}$ University of Coimbra, Portugal \\ ${ }^{3}$ University of Florida, Gainsville, USA \\ ${ }^{4}$ University of Clausthal, Germany \\ ${ }^{5}$ University of Leeds, UK
}

I $\mathrm{t}$ is now fully accepted that industrial processes involving particulate systems are in the mainstream of chemical engineering practice, research and education. A scientific approach to particle processing implies the study and development of means for characterizing particles, the primary characteristic being particle size and distribution. This paper, by members of the EFCE working party on Characterisation of Particulate Solids is intended to give an overview of the evolution of the subject and some ideas on future developments. Comparison is made of the content of the Particle Size Analysis Conference of 1970 (PSA 1970) with that of the Particulate Systems Analysis Conference held in 2003 (PSA 2003). This shows the move away from absolute methods to more rapid secondary methods such as light scattering. Further modern developments are the application of in-line measurement for understanding industrial processes and their modelling, and the growth of image-based systems and tomography applied to industrial processing. Finally there is renewed interest in methods for sub-micron particle sizing for nanotechnology.

Keywords: particle size analysis; measurement; tomography; nanoparticles; on-line methods.

\section{INTRODUCTION}

It is now fully accepted that industrial processes involving particulate systems are in the mainstream of chemical engineering practice, research and education. The roots of chemical engineering particle technology go back a long way but a significant impulsion may be attributed to the American book Micromeritics published in 1943 by J. M. Dallavalle, and the British book Small Particle Statistics published in 1960 by G. Herdan. A further impulse was given in the 1960s, when the chemical engineering departments of the then new universities of Loughborough and Bradford in Great Britain started research groups in particle technology. The action was parallelled on the European mainland by the groups of Professor Rumpf in Karlsruhe, Professor LeGoff in Nancy and Professor Heertjes in Holland. The journal Powder Technology was started in 1968 under its first editor John Williams.

*Correspondence to: Professor John Dodds, Ecole des mines d'Albi, Centre de Recherche Poudres et Procedes, Campus Jarland, Route de Teillet, 81013 Albi CT cedex, France.

E-mail: dodds@enstimac.fr

This paper is dedicated to the memory of our co-author, friend and colleague, Professor Brian Scarlett who died on 2 September 2004.
All fields of science and technology are based on the possibilities of measuring something and expressing it in numbers. The basis of a scientific approach to particle processing comes from the study and development of means for characterizing particles, the primary characteristic being particle size and particle size distribution. This led to the creation of the European Federation of Chemical Engineering Working Party on Characterization of Particulate Solids set up by its first chairman Professor Kurt Leschonski. This paper is intended to give a partial view of the evolution of the subject and some ideas from the working party on future developments.

\section{CHANGES OVER THE LAST 30 YEARS}

One of the main actions of the working party on the Characterization of Particulate Solids has been to support the PSA series of conferences. One way of marking the changes in the subject over the last 30 years is by examining the programme of The Particle Size Analysis Conference organized by the Society for Analytical Chemistry in Bradford in 1970 (Groves et al., 1972) and the recent Particulate Systems Analysis Conference organized by the Royal Society of Chemistry in Harrogate, $34 \mathrm{~km}$ distant and 33 years later in 2003. 
Table 1. Number of papers per type of system studied.

\begin{tabular}{lc}
\hline Type of system & Number of papers \\
\hline Wet & 50 \\
Dry & 30 \\
Wet or dry & 17 \\
Nanoparticles & 12 \\
\hline
\end{tabular}

\section{PSA 1970}

The Particle Size Analysis conference organized in Bradford over 3 days in 1970, was the second of its kind, the first being held in Loughborough 3 years previously. About 180 people attended to listen to 31 papers and two plenary lectures. Eight firms had stands in the exhibition.

Particle sizing in liquid suspensions was involved in two thirds of the papers (22), and of these, 15 papers dealt with sedimentation methods; mainly under gravity (12) and two on centrifugal sedimentation. The other liquid systems measurements involved the Coulter Counter (4) and only two papers involved interactions between particles and light. Theoretical papers dealt with definitions of particle size and distributions and the theory of sedimentation. Of the (9) papers on dry systems only two dealt with particle size determination; the others were concerned with surface area measurements, equally split between gas adsorption methods, air permeability methods and calorimetric methods. Sieve sizing was extensively dealt with in the two plenary lectures. Comments were also made in several sessions about short-cut and graphical methods for solving equations in cases where analytical solutions were not available. It was also mentioned that results were easier to provide in tabular form due to the time required by draughtsmen to draw graphs.

\section{PSA 2003}

The Particulate Systems Analysis conference was organized in Harrogate over three days in 2003. The aim was to re-start the PSA series of conferences, which had stopped at the 1993 conference in Loughborough. In the intervening period particle characterization had always been an important part of other events such as the World Particle Technology conferences and national and International Chemical Engineering conferences.

The change in name of the Harrogate conference indicates a change in emphasis of the subject. There were 63 papers in three parallel sessions, 25 posters, seven plenary sessions, and 24 firms had stands in the equipment exhibition, giving a good indication of the continuing interest in the subject of particle characterization. Of course the use of computers was completely taken for granted which changed entirely the methods of data collection, treatment and presentation of results with respect to 30 years pre-

Table 2. Number of papers per type of measurement.

\begin{tabular}{lc}
\hline Measurement of interest & Number of papers \\
\hline Particle size or shape & 43 \\
Bulk properties of dry powders & 17 \\
Structure or surface in wet systems & 23 \\
\hline
\end{tabular}

Table 3. Number of papers for each measuring technique.

$\left.\begin{array}{lcr}\hline \text { Measuring technique } & \text { Number of papers } & \\ \hline \text { Laser diffraction } & 10 \\ \text { Light scattering } & 11\} & 21 \\ \text { Image analysis } & 8 \\ \text { Tomography } & 15 & \\ \text { Ultrasound } & 6 \\ \text { Chromatography + FFF } & 4\} & 23 \\ \text { Sedimentation } & 1\end{array}\right)$

viously. A breakdown of the subjects dealt with is given in Tables $1-3$

Changes in emphasis in the subject can be seen by comparing the contents of PSA 1970 and PSA 2003, the first obvious change being the change in style. The 1970 conference is available as a book and includes the texts of the discussions of the papers by the delegates at the time of presentation. The 2003 conference is available as a CD with colour graphs and photos. Concerning the changes in content, particle size analysis remains the main interest in characterizing particulate system, though overall properties of powders together represent about half of the papers. Particle size analysis of dry powders, which were practically absent in 1970, now are mentioned in almost half of the papers. The most significant changes are in the types of measurement used. Measurements based on interactions between particles and light were absent in 1970, in 2003 they are by far the most used methods, especially if you include image analysis. This is undoubtedly due to the success of light diffraction methods supplanting most of the other instrumental methods of particle sizing. PSA 2003 also shows the generalization of imaging methods (image analysis and tomography) based on the increased and cheaper possibilities for rapid powerful computers. It can be seen that sedimentation methods, once preponderant are only mentioned in one paper in PSA 2003. It is tempting to say that there is a move from micron-sized systems to nano-sized particles but this is only apparent and due to new vocabulary. In PSA 1970 the analysis of sub-micron pigments, colloids and aerosols was important using centrifugal sedimentation and light diffusion techniques.

A certain number of more general differences can be identified. In PSA 1970 the systems studied were practically all dilute whereas in PSA 2003 new developments allow concentrated systems to be analysed. It may also be said that in PSA 1970 the emphasis was on laboratory analysis involving off-line measurements made using direct methods (counters, microscopy) or field classification methods (sedimentation, sieving). In PSA 2003 the emphasis is on secondary methods (laser diffraction, ultrasound attenuation) and the move of particle size analysis from the laboratory to the process plant with the use of in-line techniques.

\section{FUTURE DEVELOPMENTS}

\section{Making, Measuring, Modelling, Manipulating}

The working party on the Characterization of Particulate Solids is concerned with the industrial production of solid 
products. Products comprising a dispersed phase in a continuous phase, whether powders, pastes, slurries or aerosols, are estimated to represent $80 \%$ of the products or intermediates of the process industries. Such products are characterized by user properties which owe a lot to the structure imparted by the process used for their making. Product design and properties are thus closely linked to process design and operation. Chemical engineers are then in the 4Ms business: The Making and Manipulating of products is necessarily linked through Measuring and Modelling.

On one hand the product requires measurement of user properties involving particle size and shape distributions. On the other hand, the manufacturing process requires measurement and control. The development of in-line and in-situ measurements offers the possibility of making the link between these two which must necessarily pass by a model. One illustration of these ideas is the use of in-line laser diffraction to follow the crystal size distribution from a draft tube crystallizer. It can be seen from Figure 1 that if you get the wrong flow rate and the wrong temperature, then the process oscillates and the median crystal size varies from $500 \mu \mathrm{m}$ to $1000 \mu \mathrm{m}$ This could not be seen using conventional off line measurements. If a sample was taken over several minutes then divided to give a representative sub-sample which was analysed in the lab, we would obtain a time averaged particle size distribution very different from the real-time measurement.

Why is the crystallizer oscillating? It is because the crystals are colliding with each other, with the wall, and with the stirrer. Small crystals are broken off and act as seeds for the next generation of growth. When you have a lot of large crystals you get a lot of seeds, therefore poor growth. When you only have a few large crystals you get few seeds and excessive growth and that is why the process oscillates. Any idea that by holding the feed and process conditions constant will lead to a constant product is naive. You need to measure and model the process and properly control it. This imposes in-line or in-situ measurements with a model to link product quality (particle size distribution) to the process operation.

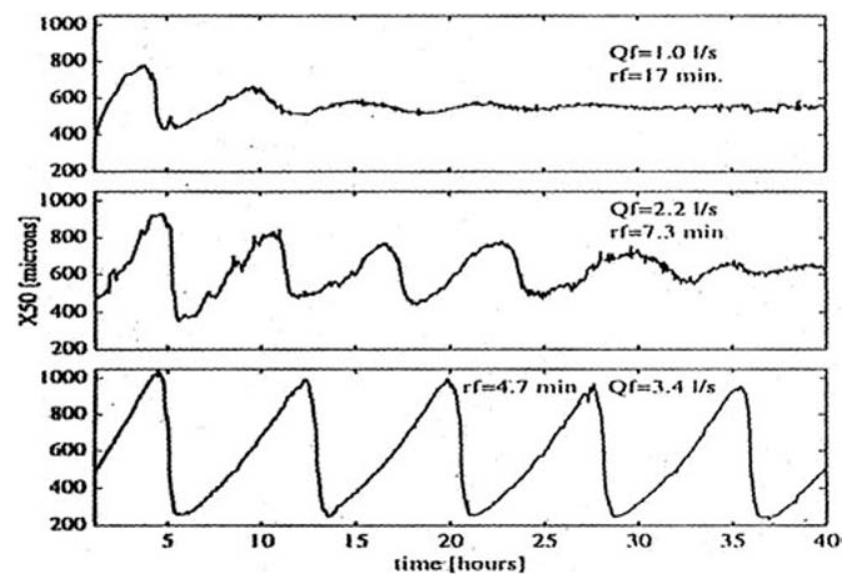

Figure 1. Median crystal size $\mathrm{X}_{50}$ from draft tube crystallizer as a function of feedrate (UNIAK Project. Laboratory for Procees Equipment, T.U. Delft)

\section{Imaging and Tomography}

Primary and secondary processing of minerals and metals involves handling materials in particulate form of variable size, shape, chemical composition and mineralogical composition. Such systems have been the testbed and a major driver for many developments in particle science over the last few decades. There has been an increasing emphasis on the use of image based methods, facilitated by the developments of digital technology. Complex mineral process systems demand careful assessment of the nature of the feedstocks and quality of the products. In De Re Metallica of 1556 Agricola describes the first recorded image-based control system based on hand sorting of ores to pick out the high grade pieces of ore from lower grade material. The use of the discerning human eye to assess colour, hue and lustre coupled with size and shape was effective as an ore-sorting and quality control mechanism - indeed the same protocol and variations on it have been in continuous use since Agricola's time. With optical sensors and discriminating intelligence beginning to emulate human capabilities, at least for the simplest of tasks, it is therefore not surprising that the minerals industries have been early adopters of process imaging. Vision-based systems based on optical sensors have been developed and deployed in industrial plant for particle sorting, on-line measurement of foams and froths, and for recognition of flows (Williams, 2005).

Since the mid-1980s the European community has been at the heart of driving the development of industrial process tomography for going beyond an external image (or snapshot) by using tomographic methods to look right into the heart of the process in three dimensions and in real time (Williams and Beck, 1995). These methods can use one or more of many sensing modalities (X-ray, electrical impedance, ultrasound, etc.) to detect differences in system properties.

Some examples of significant milestones in industrial application include: the use of electrical capacitance tomography for imaging powder flows and conveying processes (Figure 2) (Ostrowski et al., 1999, Jaworski et al., 2001); the mapping of solids, gases and fluids in tanks, reactors, crystallizers and separators (Figure 3) (Williams et al., 1999; Bennett et al., 1999); monitoring of solids deposition in pipelines and detailed vector velocity measurement via cross correlation (Figure 4) (Fangary et al., 1998; Wang et al., 2003); and finally detailed particle aggregate structures rendered using X-ray microtomography that can be used or coupled with fluid flow models, Figure 5 (Williams and Jia, 2003; Selomulya et al., 2003).

The particle technology community will continue to be pioneering in these developments with the increasingly sophisticated capabilities that can be expected to emerge in future years with advent of miniaturized sensors coupled with distributed communications systems.

\section{Nanoparticle Sizing}

The range of sciences and technologies that study particles go from astrophysics to nuclear physics. Of course, in this broad spectrum, particles are not always faced identically. The Particle Technology community deals mainly with particles from the millimetric to the nanometric region, as described in Figure 6 (Xu, 2000). 
(a)

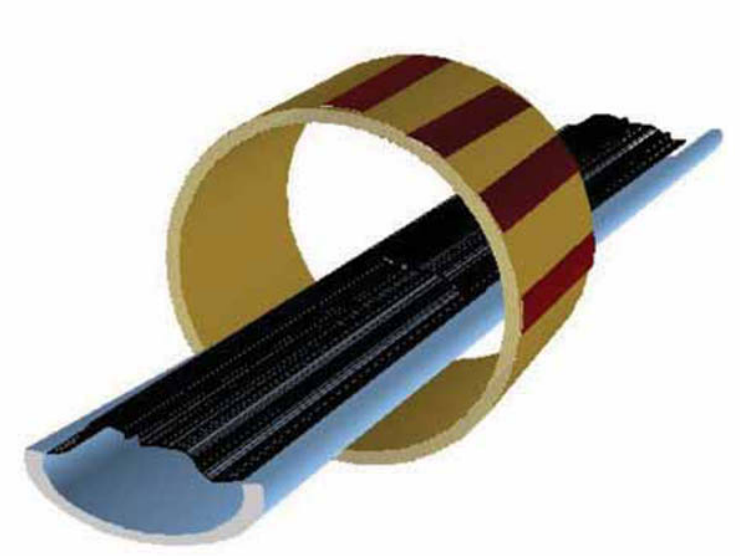

(b)
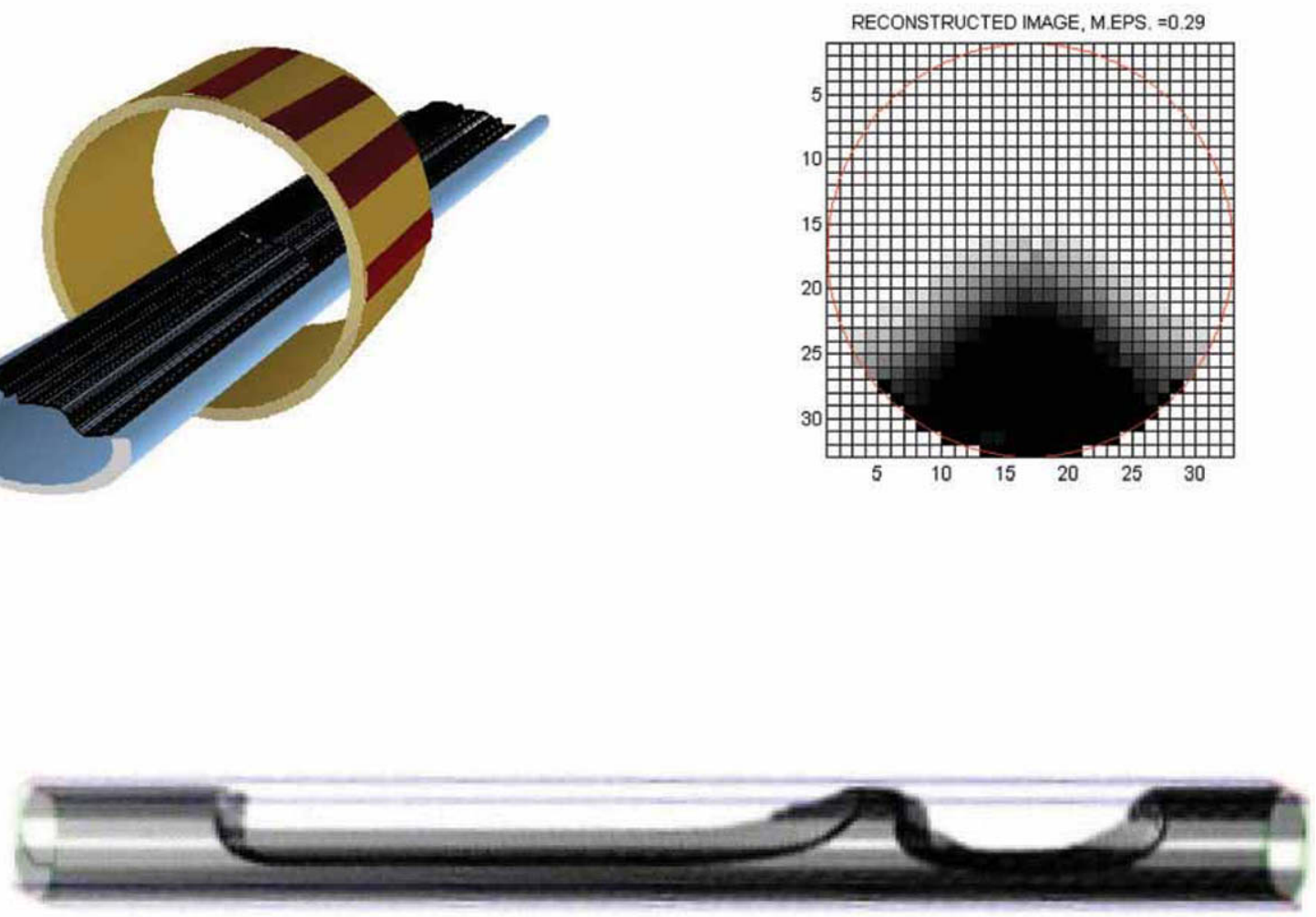

(c)

Figure 2. The use of electrical capacitance tomography for imaging powder flows and conveying processes showing here (a) sensor array around a belt conveyor, (b) cross-sectional image of particles on a belt conveyor and (c) 3D real-time measurement of slug flow in pneumatic conveying (Williams, 2005: Ostrowski et al., 1999).
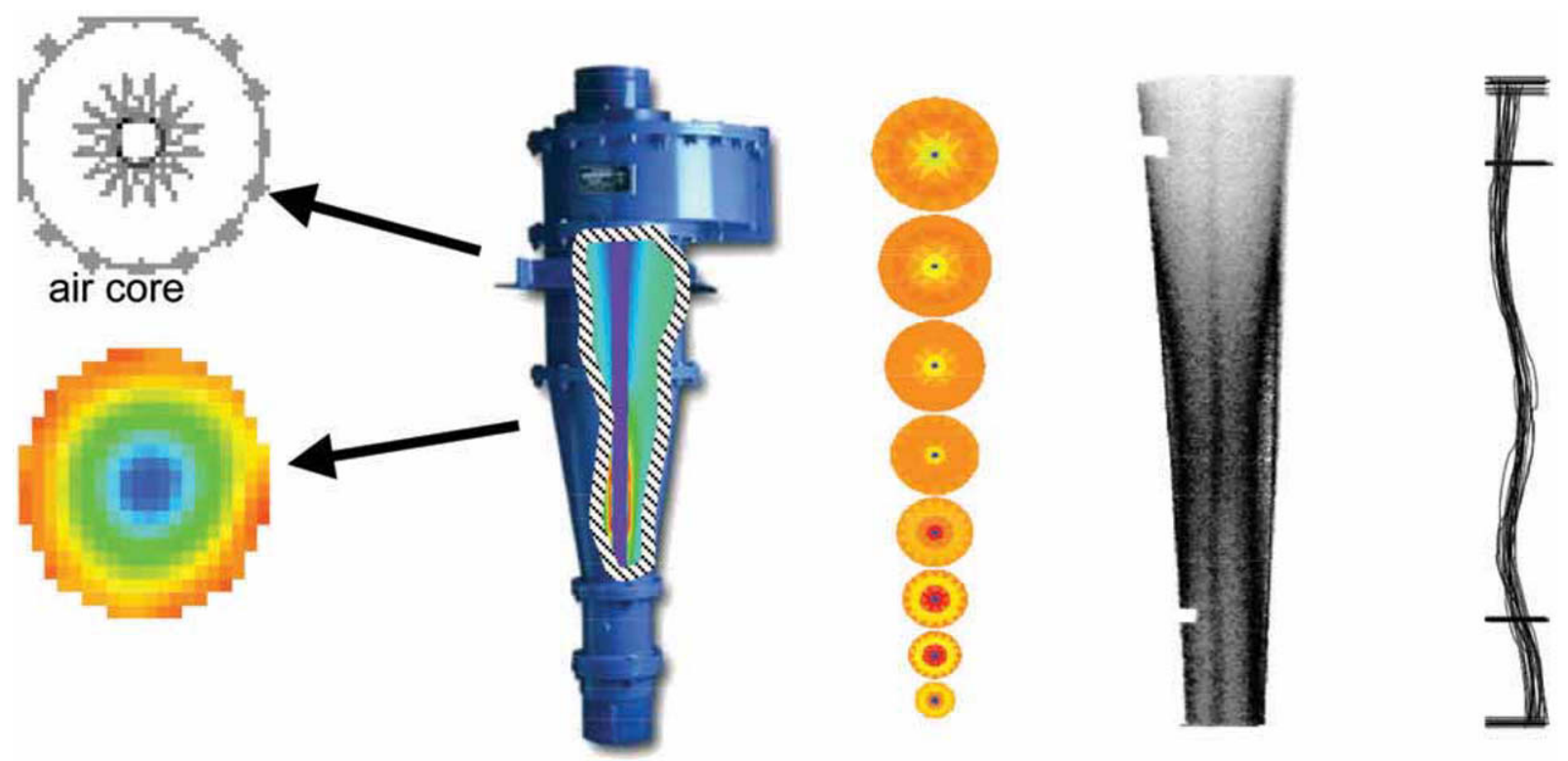

Figure 3. Use of three different tomographic methods for mapping the distribution of slurry and air core phases inside a 50 mm diameter hydrocyclone separator. Electrical impedance identifies the solids concentration and air core interface (left, bottom and middle sequence at eight different imaging planes along the cyclone). Ultrasound tomography confirms the location of the central air core at a given position along the cyclone (left top). X-ray photography provides a precise location of the asymmetric air core (far right) (Williams, 1999, Williams and Jia, 2003). 

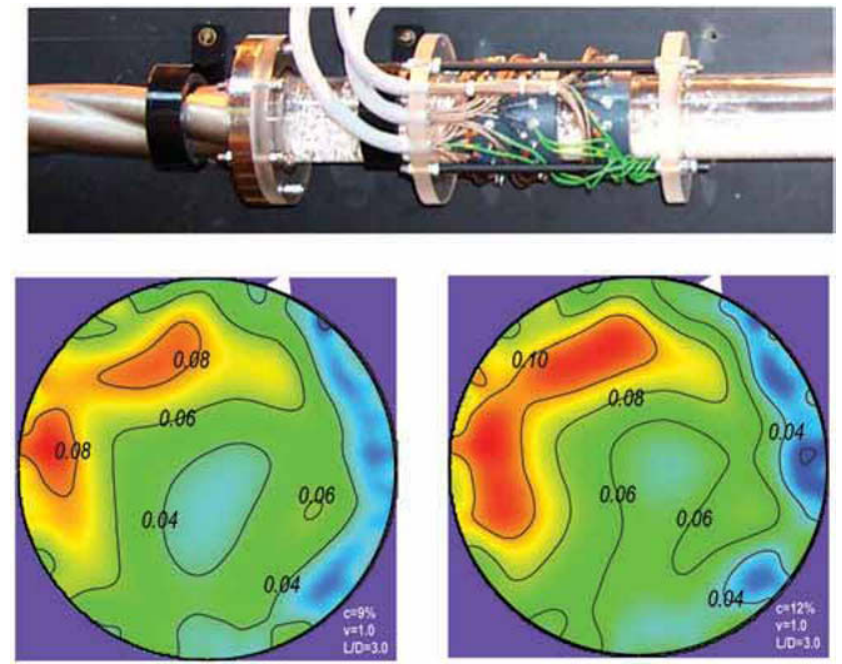

Figure 4. Monitoring solids dispersion in a strongly swirling flow pipe using electrical impedance tomography showing crescent shaped regions of high concentration $(10 \mathrm{v} / \mathrm{v} \%)$ at two measurement planes along the pipe (see photograph). Cross correlation can be used to extract velocity data (Williams, 2005, Wang et al., 2003).

Nevertheless, even in this restricted community, linked mainly to the industrial applications of particulate matter, the term particle refers to a large variety of realities: powders, emulsions, aerosols, droplets, macromolecules, proteins, enzymes and other microorganisms, etc. This means that when speaking about the characterization of particulate matter, or more generally of disperse systems, a broad range of techniques is required, since usually, it is not possible to use the same single technique to characterize both millimetric and nanometric particles. In 1981 there were already 400 different methods available for the characterization of particle size, shape and surface area (Kaye, 1982). Initially, the methods available relied mainly on the physical separation and classification of the different fractions, enabling, simultaneously, the measurement of the amount of matter in each size fraction. This was the case with the sieving and sedimentation (gravitational and centrifugal) techniques, dating back, respectively, to 1867 and to the 1922 techniques that are directed mainly to micrometric particles (sieving: 5-10 $000 \mu \mathrm{m}$, gravitational sedimentation: $2-100 \mu \mathrm{m}$ ) except for the centrifugal sedimentation technique that can go down to $0.05 \mu \mathrm{m}(50 \mathrm{~nm})$. Yet another group of techniques relies on the counting of particles, like microscopy and the electrical sensing zone technique (Coulter Counter), dating back to the 1950 s.

After 1970, other, more friendly techniques started appearing, which are mainly related to the development of laser systems, electronics and computers, such as: Optical Extinction Techniques, Laser Diffraction, Photon Correlation Spectroscopy (PCS) and other light scattering techniques not so widely spread (Van de Hulst, 1981; Pecora, 1985; Provder, 1991; Wriedt, 1998). This has allowed a quicker inspection of a larger number of particles in a non-intrusive way. Moreover, these techniques have developed further to allow inspection of either a wider range of particles or of smaller and smaller particles, in the nanometric range.

Nowadays this is extremely important, since particle technology, at the industrial level, is moving more and more into the nano range. Just think of magnetic and optical materials, microelectronics, biomaterials, cosmetics, pharmaceutical drugs and other health products, bioproducts, etc. Generally speaking, when referring to product design-a very popular subject nowadays-we are usually directed to think about some type of nanoparticles, quite often 'engineered particles', the engineer always being faced with the problem of both characterizing and handling this kind of particle.

This reality is quite obvious if we analyse the evolution of ISI references to nanoparticles from 1980 to 2000 (Stark and Pratsinis, 2002):

1980-1989: 6 references

1990-1996: 154 references

1997-2000: 1200 references

Moreover, if we search in the ISI database for entries related to nanoparticles characterization/measurement for

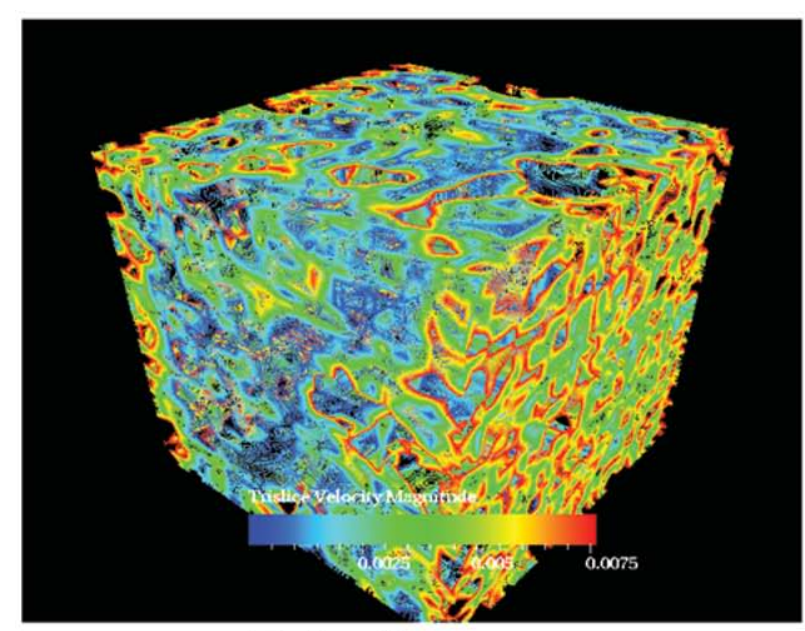

Velocity

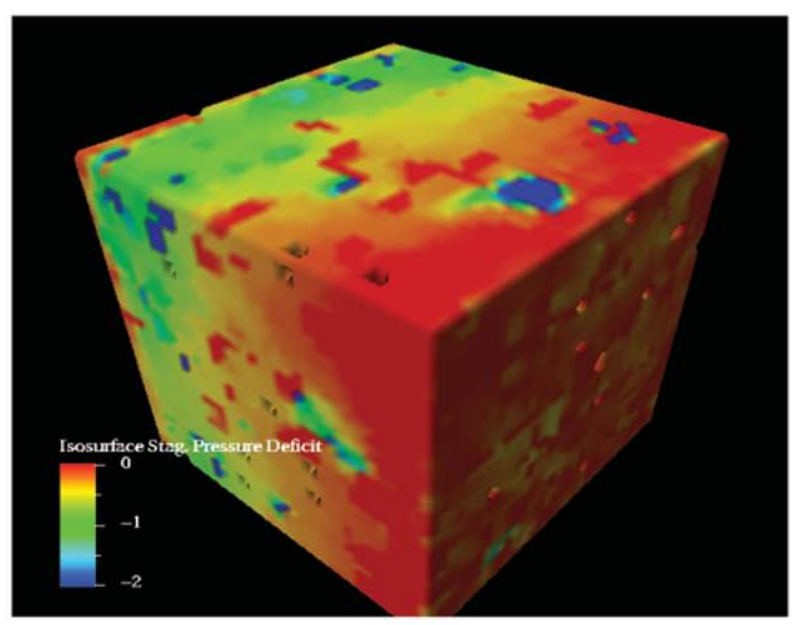

Pressure

Figure 5. Detailed 3D particle aggregate structures in a packed sediment rendered using X-ray microtomography that can be used or coupled with fluid flow (Lattice Boltzman) models to predict flow or pressure drop within the structure (Williams, 2005; Williams and Jia, 2003; Selomulya et al., 2003). 


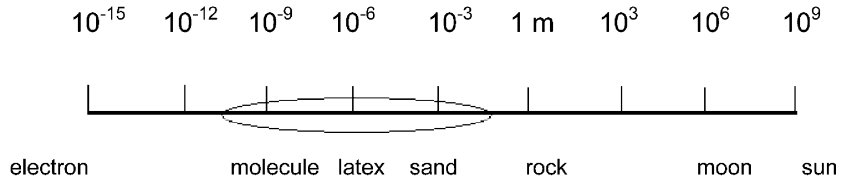

Figure 6. General size range of particles.

the 5-year period close to the first PSA conference (19701975) and to the last five years (1999-2004), the following information is retrieved:

1970-1975: 11 references

1999-2000: 103 references

clearly showing increased interest on the characterization of nanoparticles, driven by the industrial applications of these particles, though in fact, at the scientific level, there were already concerns with this area during the period 1970-1975. It must be mentioned that in the period 1970-1975 the usual expression was submicron particles rather than nanoparticles, no references being retrieved when we introduce nanoparticles as a keyword.

As far as commercial instruments are concerned, initially, the most common techniques for nanoparticles characterization were the microscopic techniques based on the bombardment of electrons (electron microscopy, EM) both transmission and scanning electron microscopes (TEM and SEM), going down to 0.001 and $0.02 \mu \mathrm{m}$, respectively. With the development of the Near Field Scanning Optical Microscopy (NSOM) (Shik and Dunn, 1999) the limit for the use of optical microscopes has been reduced to the submicron region. Moreover, Scanning Tunnelling Microscopes (STM) and Atomic Force Microscopes (AFM) have allowed an easier preparation of the samples, but the problem subsists as far as the representativity of the sample is concerned. Microscopy became more attractive during the 1980s with the appearance of commercially available image analysis systems, as a result of the advances in both computer science and electronics. Nevertheless, microscopic techniques for the nanorange are still restricted to the laboratory and mainly to research. Additionally, centrifugal techniques, which can be traced back to 1962, belong to the earlier group of techniques used to analyse nanometric particles.

Light scattering techniques have acquired an increasing importance as characterization methods since their first appearance, as commercial techniques, in the 1970s. This was made possible due to the availability of laser technologies, and again, due to the development of computing facilities and electronics. This wide acceptance is mainly due to the ease of operation and flexibility of these techniques. Initially, the first light scattering instruments to become available were based on Laser Diffraction (LDS), being restricted to micrometric particles (c. 1972) when using the Fraunhofer theory to deconvolute the signal (scattered light energy) into particle size distribution. However, modern computers have allowed the rapid processing of more complex light scattering theories, like the Mie theory, which, coupled with modern electronics, enabling signal acquisition in backscattering, and the use of more than one laser wavelength, enabled the lower LDS size limit to move down to
$0.02 \mu \mathrm{m}$ (De Boer et al., 1987; Witt and Rothele, 1996; Muller and Mehnert, 1997; Wriedt, 1998; Teipel, 2002).

On the other hand, photon correlation techniques (PCS), based on the analysis of the fluctuations of the light scattered by small particles in Brownian motion when they interfere with a light beam, are most qualified for the characterization of particles in the nanometric region $(1 \mathrm{~nm}$ to $1000 \mathrm{~nm}$ ). Photon correlation experiments were pioneered in the 1950s, but the first commercial applications date back to middle 1970s (Pecora, 1985; Rasteiro et al., 2003). More recently, photon correlation has moved into more concentrated systems by the introduction of either fibre optics or backscattering acquisition detectors, or still with the introduction of Diffusing Wave Spectroscopy-(DWS) based on the analysis of autocorrelation functions from multiple scattered light (Pine et al., 1988). In recent years ( $c$. 1997) photon migration techniques, applicable for particles larger than $100 \mathrm{~nm}$, were also introduced (Richter et al., 1998). Table 4 summarizes the light scattering characterization techniques available, quasi-elastic light scattering being, in principle, more adequate to the characterization of nanoparticles. Another light scattering technique for nanoparticles, most directed to applications in reactive stationary flows (particle formation, particle removal and combustion) with the capacity to be used on-line, is Laser Induced Incandescence (LII), the first developments dating back to 1986 (Starke et al., 2003).

In recent years, acoustic techniques have received special attention (1996 onwards) due to their ability to deal with concentrated suspensions (in some cases up to $60 \%$ vol.). These techniques are based on the attenuation of sound waves (usually ultrasound waves) as a function of their frequency, typically from 1 to $150 \mathrm{MHz}$. Acoustic spectroscopy allows the characterization of particles from 0.01 to $1000 \mu \mathrm{m}$. Electroacoustic spectral analysis allows the simultaneous determination of the zeta potential of the samples (McClements, 1996; Hackley and Texter, 1998). Light scattering techniques can also be coupled with the use of an external field, allowing the simultaneous determination of zetapotential.

Table 4. Light scattering techniques

\begin{tabular}{|c|c|c|}
\hline $\begin{array}{l}\text { Scattering } \\
\text { frequency } \\
\text { changes }\end{array}$ & Name & Applications \\
\hline \multirow[t]{10}{*}{ Elastic } & Static LS (LDS) & Sizing \\
\hline & Optical counting & Sizing and counting \\
\hline & Focused beam reflectance & Sizing \\
\hline & Time of transition & Sizing \\
\hline & Time of flight & Sizing \\
\hline & Turbidimetry & Sizing, composition study \\
\hline & Transient LS & Shape study \\
\hline & Forced Rayleigh LS & Diffusion study \\
\hline & Resonance LS & Aggregation study \\
\hline & Pulse displacement & Sizing, flow velocity study \\
\hline \multirow[t]{5}{*}{ Quasi-elastic } & PCS/DWS & Sizing and diffusion study \\
\hline & Electrophoretic LS & $\begin{array}{l}\text { Mobility, sizing and } \\
\text { diffusion study }\end{array}$ \\
\hline & Phase doppler & $\begin{array}{l}\text { Sizing, counting, flow } \\
\text { velocity study }\end{array}$ \\
\hline & Photon migration & Sizing \\
\hline & $\begin{array}{l}\text { Modulated } \\
\text { dynamic LS }\end{array}$ & $\begin{array}{l}\text { Particle mass, diffusion, } \\
\text { velocity and shape study }\end{array}$ \\
\hline Inelastic & RCS (Raman scattering) & Composition \\
\hline
\end{tabular}


In the nanometric region, chromatographic techniques have long been considered as particle characterization techniques (1979). Size Exclusion Chromatography (SEC) allows both the fractionation and sizing of nanoparticles $(0.001-0.5 \mu \mathrm{m})$ being used for both nanoparticles and macromolecules (Yau et al., 1979). More recently (1982), Hydrodynamic Chromatography (HDC) was introduced, covering the range $0.02-50 \mu \mathrm{m}$. In this case, the particles are fractionated due to the velocity profile developed in the capillary column and to the interaction between the particles and the liquid flow profile (Provder, 1991). These techniques require always an a priori calibration. Field flow fractionation uses also the chromatographic principle to fractionate nanoparticles, but the fractionation is further enhanced by the superimposition of an external field. This idea dates back to the 1960s. In Sedimentation Field Flow Fractionation $\left(\mathrm{SF}^{3}\right)$ the superimposed field is a centrifugal field (Giddings, 1966). Equipment applying this principle was commercialized in the 1990s but it never became popular and the main application is still related to research. The latest particle technology conferences (2003-2004) have shown a large increase of the number of scientific papers dealing with chromatographic techniques, the same applying to the number of commercial equipment being displayed.

Other recent techniques directed to the characterization of nanoparticles that were presented in the last decade of particle technology conferences are: dielectric spectroscopy for liquid born colloids (Miller et al., 1995) and the Pulsed Field Gradient NMR (PFG-NMR), 1996, which determines the diffusion coefficient for particles (micelles and latexes) up to $100 \mathrm{~nm}$ (Blees et al., 1996).

To finalize, it can be said that the spread of the use of nanoparticles is strongly dependent on the ability to measure several physical and chemical properties of the particles in a reproducible and accurate way. This has clearly resulted in the need for development of new tools, techniques and standards for the nanometric size range (Jillavenkatesa and Kell, 2002). Moreover, the increased surface to volume area in these systems leads to particle interactions that may not be significant in coarser powders. Thus, another concern that has driven recent research is the need to move to the analysis of more concentrated systems, since dilution can affect those interaction mechanisms (Lehner et al., 1998; Jiang, 1998). Therefore, in many cases, it can also be important to have the possibility of distinguishing between primary particles and aggregates.

Another trend is, as happened earlier for the larger particles, the need to go on-line in the process to characterize particles in the nanometric region. This is, altogether, more important, since one is usually dealing, in this case, with high added value products. This need will increasingly demand that the nanoparticle characterization instruments possess features such as ease of operation, robustness, rapid results supplying capacity and the capability to operate in severe environments. These characteristics have to be coupled with the capacity to produce in-process robust sensors for control applications as well as miniature sensors. Eventually, these processes will require the development of characterization platforms using optical, acoustic and chemical sensors. Moreover, the concept of virtual measurements and virtual laboratories is appearing as an emergent methodology in relation with nanotechnology and nanoparticles processes (Trichard, 1999).

\section{CONCLUSION}

New methods of measurement are always the precursor to new developments in technology. We hope that this article will go some way to illustrating that the theory and practice of industrial processes involving particles has been transformed in the last decades by the availability of particle characterization methods. These are no longer only for particle size analysis in a laboratory but also for composition, structure and user properties of systems composed of particulate solids in industrial plants. These new methods are especially interesting for on-line measurements, imaging techniques and methods for nanoparticle measurements.

\section{REFERENCES}

Agricola, G., 1556, De Re Metallica translated by H.C. Hoover and L.H. Hoover (Dover, New York, USA: 1950, 638 pp).

Bennett, M.A., West, R.M., Luke, S.P., Jia, X. and Williams, R.A., 1999, Measurement and analysis of flows in a gas-liquid column reactor, Chem Eng Sci, 54(21): 5003-5012.

Blees, M.H., Geurts, J.M. and Leyte, J.C., 1996, Self-diffusion of charged polybutadiene latex particles in water measured by pulsed field gradient NMR, Langmuir, 12: 1947-1957.

Dallavalle, J.M., 1948, Micromeritics, (1st edition 1943, 2nd edition, Pittman).

De Boer, G.B.J., de Weerd, C., Thoenes, D. and Goossens, H.W.J., 1987, Laser diffraction spectroscopy: Fraunhofer diffraction versus Mie scattering, Part Charact, 4: 14-19.

Fangary, Y.S., Williams R.A., Neil W.A., Luke S.P., Bond, J. and Faulks, I., 1998, Application of electrical resistance tomography to detect deposition in hydraulic conveying systems, Powder Technology, 95: $61-66$.

Giddings, J.C., 1966, A new separation concept based on a coupling of concentration and flow nonuniformities, Sep Sci. 1: 123-125.

Groves, M.J. and Wyatt-Sargent, J.L., 1972, Particle Size Analysis 1970 (Society of Analytical Chemistry, London, UK).

Hackley, V.A. and Texter, J. 1998, Handbook on Ultrasonic and Dielectric Characterisation Techniques for Suspended Particulates. (ACS, USA).

Herdan, G., 1960, Small Particle Statistics (Butterworths, London, UK).

Jaworski, A. and Dyakowski, T., 2001, Application of electrical capacitance tomography for gas-solid flow characterisation in a pneumatic conveying system, Measurement Sci Technol, 12: 1109-1119.

Jiang, H. 1998, Enhanced photon-migration methods for particle sizing in concentrated suspensions, AIChE J., 44(8): 1740-1744.

Jillavenkatesa, A.H., and Kell, J.F., 2002, Nanopowder characterization: Challenges and future directions, J. Nanoparticle Res, 4: 463-468.

Kaye, B.H., 1982, in Particle Size Analysis 1981, Stanley-Wood, N., Allen, T., (eds) (J. Wiley \& Sons, New York, USA pp. 3).

Lehner, D., Kellner, G., Schnablegger, H. and Glatter, O., 1998, Static light scattering in dense colloidal systems: New instrumentation and experimental results, J Colloid \& Interface Sci., 201: 34-47.

McClements, D.J., 1996, Principles of ultrasonic droplet size determination in emulsions, Langmuir, 12: 3454-346.

Miller, J.F., Velev, O., Wu, S.C.C. and Ploehn, H.J., 1995, A combined instrument for phase analysis light scattering and dielectric spectroscopy, J Colloid \& Interface Sci, 174: 490-499.

Muller, R.H. and Mehnert, W., 1997, eds. Particle and Surface Characterisation Methods (Medpharm Sci. Pub., Stuttgart, Germany).

Ostrowski, K.L., Luke, S.P., Bennett, M.A. and Williams, R.A., 1999, Real time visualisation and analysis of dense phase powder conveying, Powder Technology, 102: 1-13.

Pecora, R., 1985, Dynamic Light Scattering: Applications of Photon Correlation Spectroscopy (Plenum Press, New York, USA).

Pine, D.J., Weitz D.A., Chaikin, P.M., and Herbolzheimer, E., 1988, Diffusing-wave spectroscopy, Phys Rev Letters, 60: 1134-1137.

Provder, T., 1991, Particle Size Distribution II, ACS Symposium Series 472. American Chemical Society, USA). 
Rasteiro, M.G., Lemos, C.C. and Vasquez, A., 2003, Extending PCS to characterise complex nanoparticles, in Proc. ECCE2003, Granada, Spain, 10.3-018

Richter, S.M., Shinde, R.R., Balgi, G.V. and Sevick-Muraca, E.M., 1998, Particle sizing using frequency domain photon-migration, Part \& Part Systems Charact, 15: 9-15.

Selomulya, C., Jia, X. and Williams, R.A., 2003, Tomographic analysis of flocs and flocculating suspensions, 3rd World Congress on Process Tomography, Banff. Manchester: VCIPT, 2003, 145-151.

Shik, H. and Dunn, R.C., 1999, Near-field scanning optical microscopy, Annal Chem, 71: 23A-28A.

Stark, W.J. and Pratsinis, S.E., 2002, Aerosol flame reactors for manufacture of nanoparticles, Powder Technology, 126: 103-108.

Starke, R., Kock, B. and Roth, P., 2003, Nano-particle sizing by laserinduced-incandescence in a shock wave reactor, Shock Waves, 12: $351-360$.

Taipel, U., 2002, Problems in characterizing transparent particles by laser light diffraction spectrometry, Chem Eng Technol, 25(1): 13-21.

Trichard, T., 1999, Virtual measurements, Res Dev, 41(6): 30.

Van de Hulst, H.C., 1981, Light Scattering by Small Particles (Dover Pub., New York, USA.).

Wang, M., Jones, T.F. and Williams R.A., 2003, Visualisation of asymmetric solids distribution in horizontal swirling flows using electrical resistance tomography, Chemi Eng Res Desi, 81(A): 854-861.
Williams, R.A., 2005, Chapter 11: Mineral and material processing, in Process Imaging and Control, Scott, D.M. and McCann, H. (eds). (CRC Press, in press)

Williams, R.A. and Beck, M., 1995, Process Tomography- Principles, Techniques and Applications (Butterworth-Heinemann, Oxford, UK, pp 550).

Williams, R.A. and Jia, X., 2003, Tomographic analysis of particulate systems, Advanced Powder Technology, 14, 1: 1-16.

Williams, R.A., Jia, X., West, R.M., Wang, M., Cullivan, J.C., Bond J., Faulks, I., Dyakowski, T., Wang, S.J., Climpon, N., Kostuch, J.A. and Payton, D., 1999, Industrial monitoring of hydrocyclone operation using electrical resistance tomography. Minerals Eng 12(10): 1245-1252.

Witt, W. and Rothele, S., 1996, Laser diffraction unlimited? Part \& Part Systems Charact, 13: 280-286.

Wriedt, T., 1998, A review of elastic light scattering theories, Part \& Part Systems Charact, 15: 67-74.

$\mathrm{Xu}$, R., 2000, Particle Characterization: Light Scattering Methods (Kluwer Ac. Pub., USA).

Yau, W.W., Kirkland, J.J. and Bly, D.D., 1979, Modern Size Exclusion Chromatography, (J. Wiley \& Sons, New York, USA.)

The manuscript was received 14 June 2004 and accepted for publication after revision 28 October 2004. 